\title{
The Type 2 Deiodinase Thr92Ala Polymorphism Is Associated with Worse Glycemic Control in Patients with Type 2 Diabetes Mellitus: A Systematic Review and Meta-Analysis
}

\author{
Xiaowen Zhang, Jie Sun, Wenqing Han, Yaqiu Jiang, Shiqiao Peng, \\ Zhongyan Shan, and Weiping Teng
}

The Endocrine Institute and The Liaoning Provincial Key Laboratory of Endocrine Diseases, Department of Endocrinology
and Metabolism, The First Hospital of China Medical University, No. 155 Nanjing North Street, Shenyang, Liaoning, China

Correspondence should be addressed to Weiping Teng; twp@vip.163.com

Received 26 April 2016; Revised 27 June 2016; Accepted 24 August 2016

Academic Editor: Ponnusamy Saravanan

Copyright (C) 2016 Xiaowen Zhang et al. This is an open access article distributed under the Creative Commons Attribution License, which permits unrestricted use, distribution, and reproduction in any medium, provided the original work is properly cited.

Objective. Type 2 deiodinase (Dio2) is an enzyme responsible for the conversion of T4 to T3. The Thr92Ala polymorphism has been shown related to an increased risk for developing type 2 diabetes mellitus (T2DM). The aim of this study is to assess the association between this polymorphism and glycemic control in T2DM patients as marked by the HbA1C levels. Design and Methods. The terms "rs225014," "thr92ala," "T92A," or "dio2 a/g" were used to search for eligible studies in the PubMed, Embase, and Cochrane databases and Google Scholar. A systematic review and meta-analysis of studies including both polymorphism testing and glycated hemoglobin (HbAlC) assays were performed. Results. Four studies were selected, totaling 2190 subjects. The pooled mean difference of the studies was $0.48 \%$ (95\% CI, $0.18-0.77 \%$ ), indicating that type 2 diabetics homozygous for the Dio2 Thr92Ala polymorphism had higher HbA1C levels. Conclusions. Homozygosity for the Dio2 Thr92Ala polymorphism is associated with higher HbA1C levels in T2DM patients. To confirm this conclusion, more studies of larger populations are needed.

\section{Introduction}

Type 2 deiodinase (Dio2) is localized on the long arm of the 14th human chromosome in 14q24.3. It is an intracellular enzyme which catalyzes the conversion of thyroxine (T4) to its active form triiodothyronine (T3) [1]. Therefore, Dio2 is a very important regulator for tissue specific metabolic activity.

A single nucleotide polymorphism in the Dio2 gene (A/G) results in a threonine change to alanine (Thr92Ala) at codon 92 [2]. The Dio2 Thr92Ala polymorphism has been reported to be associated with many disorders, such as osteoarthritis [3, 4], hypertension [5], Graves' disease [6], Kashin-Beck disease [7], bipolar disorder [8], depression [9], and cognitive impairment $[10,11]$. In addition, many studies have shown that the Thr92Ala polymorphism is related to type 2 diabetes mellitus (T2DM), insulin resistance, and body mass index (BMI) [2, 12-18]. However, the relationship between the Thr92Ala polymorphism and glycemic control in T2DM patients is unclear. The aim of this meta-analysis is to determine whether the Dio2 Thr92Ala polymorphism is associated with glycemic control in T2DM patients as marked by the $\mathrm{HbAlC}$ level.

\section{Design and Methods}

2.1. Search Strategy. A literature search was conducted by two investigators (Xiaowen Zhang and Jie Sun) in February 2016, using the PubMed, Embase, and Cochrane databases and Google Scholar. The terms Thr92Ala, T92A, rs225014, and Dio2 a/g were used. Reference lists in the articles and recent reviews were also searched.

2.2. Inclusion Criteria. Studies were selected for metaanalysis if they met the following criteria: (1) being observational studies related to Dio2 polymorphism and (2) including T2DM patients along with their respective glycated hemoglobin ( $\mathrm{HbA1C}$ ) levels. 
2.3. Data Extraction. The following data were extracted from selected studies: name of first author, year of publication, country, age, gender, duration of diabetes, treatment, population size, and HbA1C means and standard deviations (SD) of each genotype. The difference between genotypes was analyzed in both recessive and dominant inheritance model.

2.4. Quality Assessment. Two investigators (Yaqiu Jiang and Wenqing Han) assessed the quality of the selected studies using the Newcastle-Ottawa Scales (NOS) [19]. Information regarding selection, comparability, and exposure was evaluated for each study. Only the studies with more than 5 stars based on the NOS scale were included in this meta-analysis.

2.5. Statistical Analyses. This systematic review was conducted according to the recommendations outlined in the PRISMA (Supplementary Material available online at http://dx.doi.org/10.1155/2016/5928726). Review Manager 5.3 was used for data analysis. The mean differences and the 95\% confidence intervals (95\% CI) for each study and for the pooled effect were calculated to evaluate the relationship between Thr92Ala polymorphism and HbA1C value in T2DM patients. Heterogeneity was assessed using the $Q$ statistical test and $I^{2}$ statistic. A sensitivity analysis was performed by sequential omission of each study. Subgroup analysis was processed to investigate heterogeneity in the meta-analysis.

\section{Results}

Ninety-five studies were initially retrieved from the databases and Google Scholar. Seventy-three articles were excluded after reading the titles and abstracts as they were not related to T2DM. A full-text review of the remaining 22 articles was conducted and 4 articles were identified as eligible (Figure 1). Table 1 summarized the characteristics of the selected articles.

When analyzed in a recessive inheritance model, the meta-analysis of 4 studies including data on the association between Thr92Ala polymorphism and HbA1C levels showed that the mean difference (MD) was $0.48 \%$ (95\% CI, 0.18 $0.77 \%$ ) (Figure 2), indicating that the HbA1C levels in T2DM patients with Dio2 Thr92Ala homozygosity are $0.48 \%$ higher than the non-Ala T2DM patients. Both the $Q$ statistical test $(P=0.10)$ and $I^{2}$ statistic $\left(I^{2}=52 \%\right)$ showed moderate heterogeneity; therefore, a random effects model was used. Sensitivity analyses by sequential omission of each study resulted in similar results, with calculated MDs of $0.44 \%(95 \%$ CI, 0.11 0.76\%), 0.60\% (95\% CI, 0.30 0.89\%), 0.33 (95\% CI, $0.09 \sim 0.57 \%$ ), and $0.55 \%$ (95\% CI, $0.07 \sim 1.02 \%)$, respectively.

When analyzed in a dominant inheritance model, one study was excluded because Thr/Thr and Ala/Thr genotypes were grouped and compared with Ala/Ala genotype [14]. The meta-analysis of the remaining 3 studies showed that the mean difference (MD) was $0.05 \%$ (95\% CI, $-0.16 \sim 0.26 \%$ ) (Figure 3), indicating that HbA1C levels of T2DM patients with Dio2 Thr92Ala allele were not higher. Both the $Q$ statistical test $(P=0.69)$ and $I^{2}$ statistic $\left(I^{2}=0 \%\right)$ showed no heterogeneity; therefore, a fixed effects model was used.

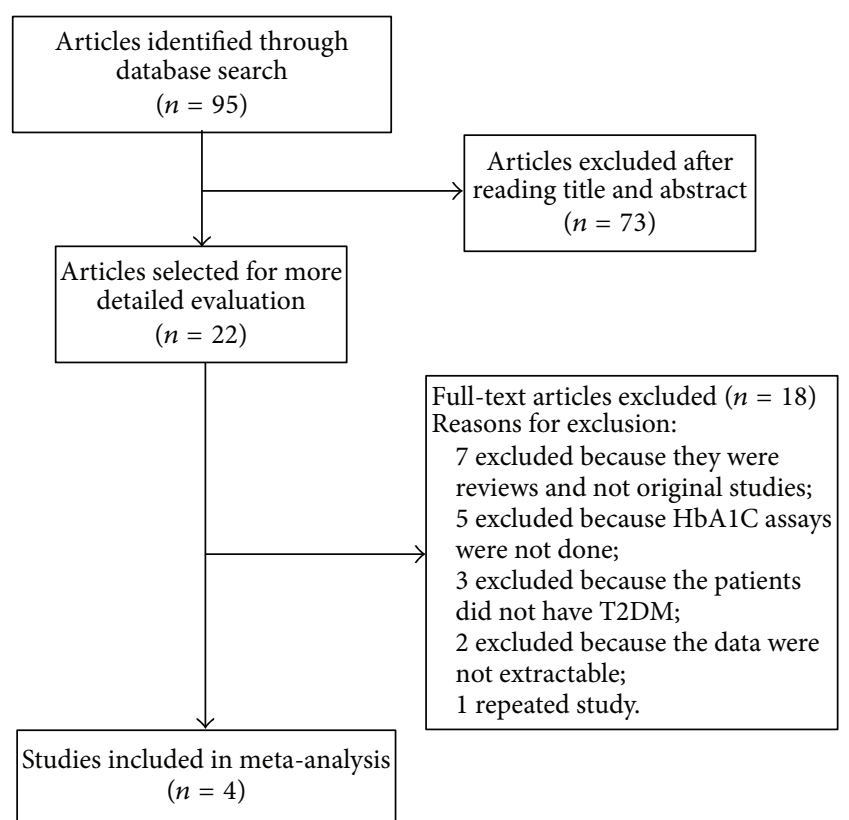

FIGURE 1: Flowchart of search results for meta-analysis.

Sensitivity analyses by sequential omission of each study resulted in similar results, with calculated MDs of $0.06 \%(95 \%$ CI, $-0.16 \sim 0.29 \%),-0.01 \%$ (95\% CI, $-0.26 \sim 0.24 \%$ ), and $0.13 \%$ (95\% CI, $-0.20 \sim 0.45 \%$ ), respectively.

In order to uncover the source of heterogeneity, subgroup analysis was performed (Figure 4). Dhanunjaya's study is different from the other 3 studies because the BMI is smaller and racial population is Indian. We found that this study was the main source of heterogeneity.

\section{Discussion}

The results of the previous studies regarding the association between the Dio2 Thr92Ala polymorphism and glycemic control in T2DM patients have been contradictory $[2,14,20$, 21]. This current meta-analysis found that people who are homozygous for Thr92Ala had 4.8\% higher HbA1C levels, suggesting that Thr92Ala homozygosity is associated with worse glycemic control in T2DM patients. To our knowledge, this is the first meta-analysis to investigate the effect of Dio2 Thr92Ala polymorphism on HbA1C levels in T2DM patients.

Some studies assumed that it is a recessive inheritance model because of "Thr/Thr and Thr/Ala genotypes showing similarities in biochemical characteristics" $[14,20]$. However, we believe that further studies are still needed and analyses performed in dominant inheritance model are necessary. There are no significant differences in Ala/Ala + Ala/Thr versus Thr/Thr when it was analyzed in a dominant inheritance model.

Among the four included studies, three were conducted in Brazil, thus related to Brazilian population [2, 14, 21], and one was done in India [20]. In the Brazilian population, the Ala allele frequency is about 0.4 ; however, in the Indian population, the frequency of this allele is 0.6 , which is 


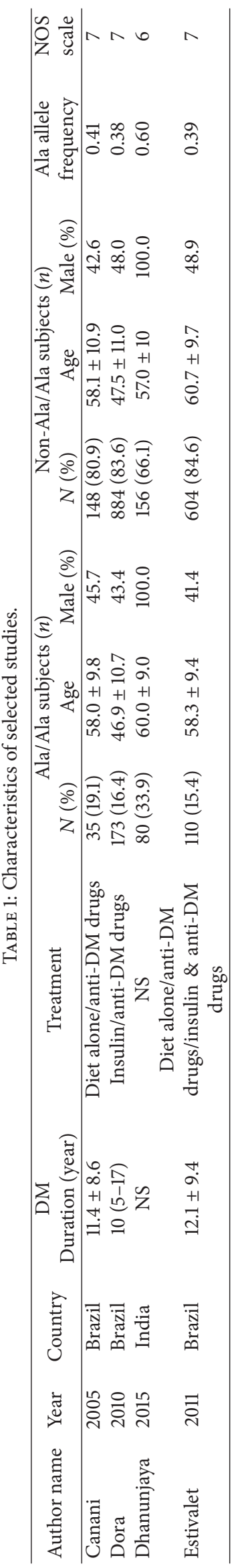




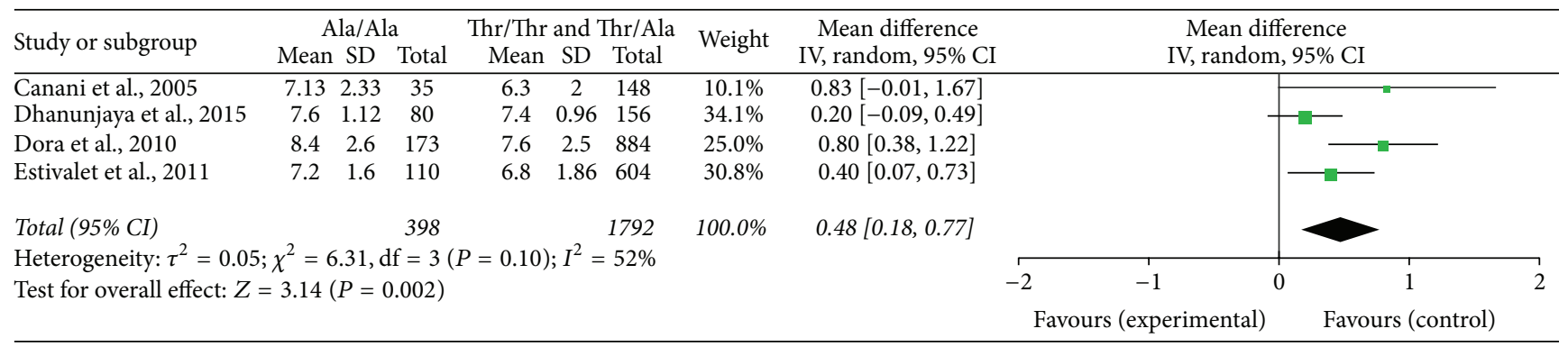

FIGURE 2: An analysis in recessive inheritance model. Individual and pooled MDs and 95\% Cl estimate for HbA1C levels in type 2 diabetes in association with Thr92Ala homozygosity of type 2 deiodinase. CI: confidence interval; HbA1C: glycated hemoglobin; MD, mean differences.

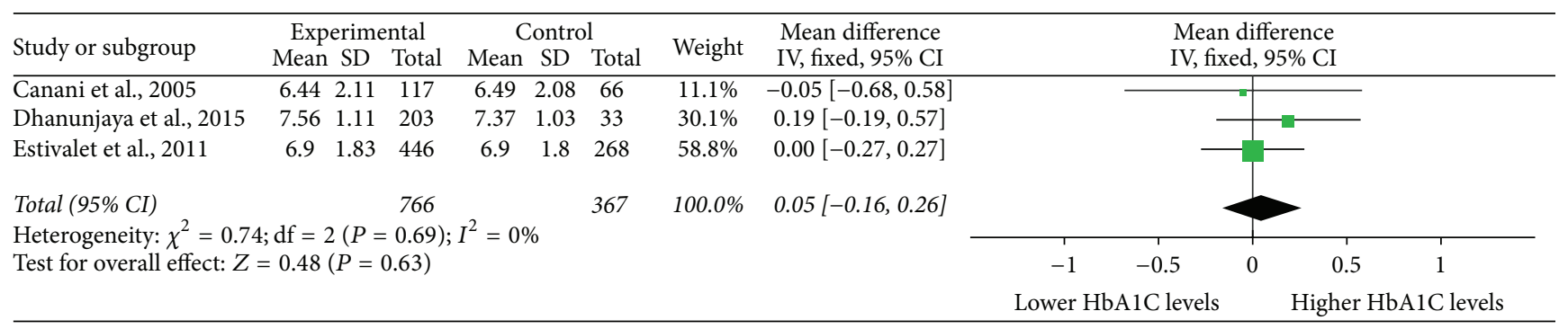

FIgURE 3: An analysis in dominant inheritance model. Individual and pooled MDs and 95\% Cl estimate for HbAlC levels in type 2 diabetes in association with Thr92Ala homozygosity of type 2 deiodinase. CI: confidence interval; HbA1C: glycated hemoglobin; MD, mean differences.

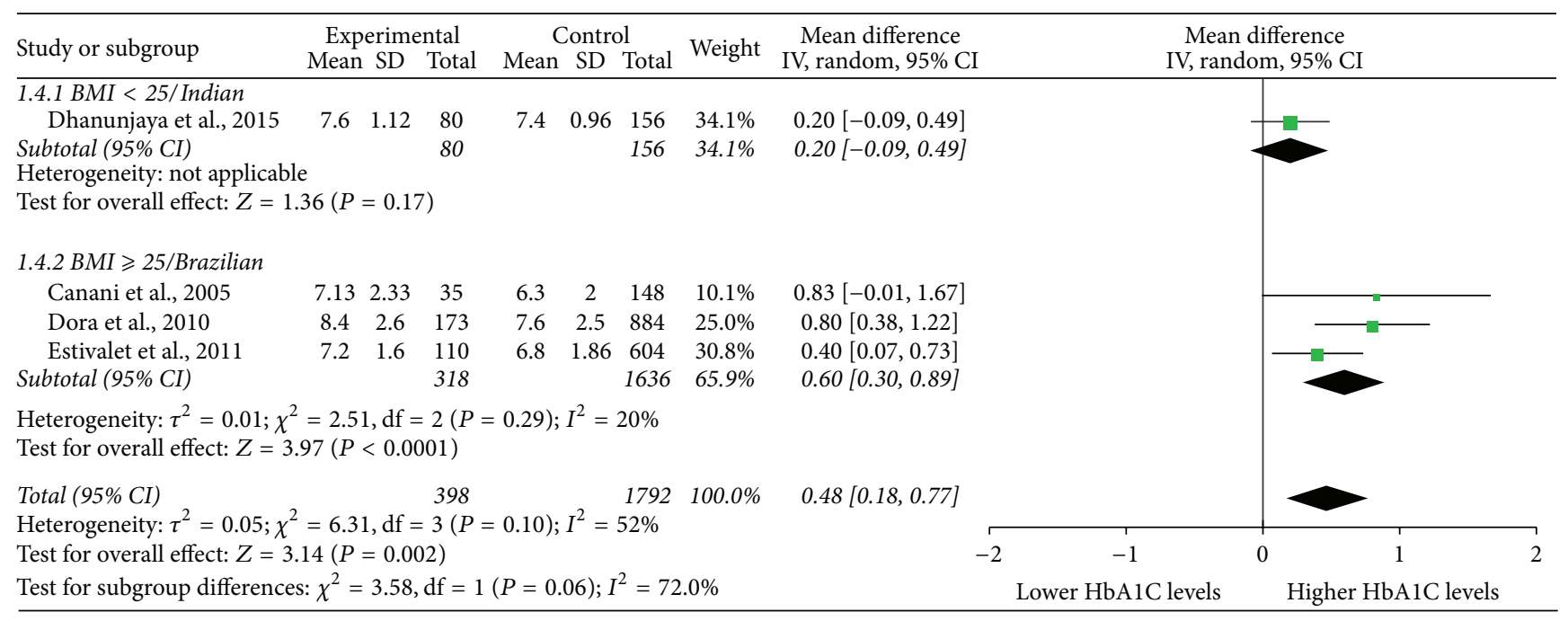

FIGURE 4: Subgroup analysis. Individual and pooled MDs and 95\% Cl estimate for HbA1C levels in type 2 diabetes in association with Thr92Ala homozygosity of type 2 deiodinase. CI: confidence interval; HbAlC: glycated hemoglobin; MD, mean differences.

much higher than that among the Brazilian population. It has been reported that the Ala allele frequencies vary among different populations: Indians (0.61), Han Chinese in Beijing (0.58), Mexican-Americans (0.54), African American (0.51), British (0.34), and Finnish (0.24) (https: //www.ncbi.nlm.nih.gov/variation/tools/1000genomes/).

Subgroup analysis shows that Dhanunjiya's study, which was different in BMI or race from the other 3 studies, would be the reason for moderate heterogeneity. Both $\mathrm{BMI}$ and race might have apotential impact on the HbA1C levels in T2DM patients. Further studies are needed to investigate these potential influential factors.

Carriers of the Ala allele have been shown to have a net decrease in glucose disposal [22]. The Dio2 Thr92A and peroxisome proliferator-activated receptor-gamma2 (PPAR $\gamma 2$ ) Pro2Ala polymorphisms interact in the modulation of systolic and diastolic blood pressure and metabolic syndrome [23]. Furthermore, a combination of this polymorphism and 
B3-adrenergic receptor Trp63Arg results in an increased BMI, indicating a synergistic effect of these two polymorphisms [13].

Some studies have shown that the Thr92Ala polymorphism is related to type 2 diabetes mellitus (T2DM), insulin resistance. However, the Framingham Heart Study showed that there is no association of Thr92Ala polymorphism with T2DM or hypertension risks $[17,24]$. Though Dio2 velocity was decreased in the thyroid and skeletal muscle in individuals homozygous for the Ala allele [2], the Thr to Ala substitution at codon 92 is not near the enzyme's active site [25]. Moreover, the Thr92Ala mutation does not affect Dio2 activity in vitro, indicating that Thr92Ala may be a marker, rather than a functional polymorphism of the decreased enzyme velocity.

The limitations of this meta-analysis are as follows: (1) T2DM is a heterogeneous disease [26], and thus the contribution of a single gene may be restricted; (2) the size of subjects involved in this meta-analysis was relatively small; (3) only Brazilian and Indian population were included and these findings may not be applied to other racial groups. More studies on this topic are needed since genetic association studies should include larger populations in order to ensure accurate conclusions.

\section{Conclusion}

In conclusion, homozygosity for the Dio2 Thr92Ala polymorphism might be associated with worse glycemic control in type 2 diabetics as marked by the HbAlC levels. Further epidemiological studies of larger populations from different geographic regions are needed.

\section{Competing Interests}

The authors declare that there are no competing interests regarding the publication of this paper.

\section{Acknowledgments}

This work was supported by Research Foundation, Department of Science and Technology, Liaoning Province Government, China (Grant no. 2011225023) and Important Platform of Science and Technology for Universities in Liaoning Province (Grant no. 16010).

\section{References}

[1] A. C. Bianco, D. Salvatore, B. Gereben, M. J. Berry, and P. R. Larsen, "Biochemistry, cellular and molecular biology, and physiological roles of the iodothyronine selenodeiodinases," Endocrine Reviews, vol. 23, no. 1, pp. 38-89, 2002.

[2] L. H. Canani, C. Capp, J. M. Dora et al., "The type 2 deiodinase A/G (Thr92Ala) polymorphism is associated with decreased enzyme velocity and increased insulin resistance in patients with type 2 diabetes mellitus," Journal of Clinical Endocrinology and Metabolism, vol. 90, no. 6, pp. 3472-3478, 2005.
[3] J. Loughlin, "Genetic contribution to osteoarthritis development: current state of evidence," Current Opinion in Rheumatology, vol. 27, no. 3, pp. 284-288, 2015.

[4] I. Meulenbelt, J. L. Min, S. Bos et al., "Identification of DIO2 as a new susceptibility locus for symptomatic osteoarthritis," Human Molecular Genetics, vol. 17, no. 12, pp. 1867-1875, 2008.

[5] O. Gumieniak, T. S. Perlstein, J. S. Williams et al., "Ala92 type 2 deiodinase allele increases risk for the development of hypertension," Hypertension, vol. 49, no. 3, pp. 461-466, 2007.

[6] B. Alina, P. Daria, F. Olga, S. Vladislav, K. Anna, and G. Elena, "Thr92Ala polymorphism of human type 2 deiodinase gene (hD2) affects the development of Graves' disease, treatment efficiency, and rate of remission," Clinical and Developmental Immunology, vol. 2012, Article ID 340542, 5 pages, 2012.

[7] L. Huang, Y. Shi, F. Lu et al., "Association study of polymorphisms in selenoprotein genes and kashin-beck disease and serum selenium/iodine concentration in a tibetan population," PLoS ONE, vol. 8, no. 8, Article ID e71411, 2013.

[8] B. He, J. Li, G. Wang et al., "Association of genetic polymorphisms in the type II deiodinase gene with bipolar disorder in a subset of Chinese population," Progress in NeuroPsychopharmacology and Biological Psychiatry, vol. 33, no. 6, pp. 986-990, 2009.

[9] E. Gałecka, M. Talarowska, A. Orzechowska, P. Górski, M. Bieńkiewicz, and J. Szemraj, "Association of the DIO2 gene single nucleotide polymorphisms with recurrent depressive disorder," Acta Biochimica Polonica, vol. 62, no. 2, pp. 297-302, 2015.

[10] M. Luo, X. H. Zhou, T. Zou, K. Keyim, and L. M. Dong, "Type II deiodinase polymorphisms and serum thyroid hormone levels in patients with mild cognitive impairment," Genetics and Molecular Research, vol. 14, no. 2, pp. 5407-5416, 2015.

[11] T.-W. Guo, F.-C. Zhang, M.-S. Yang et al., "Positive association of the DIO2 (deiodinase type 2) gene with mental retardation in the iodine-deficient areas of China," Journal of Medical Genetics, vol. 41, no. 8, pp. 585-590, 2004.

[12] S. Nair, Y. L. Muller, E. Ortega, S. Kobes, C. Bogardus, and L. J. Baier, "Association analyses of variants in the DIO2 gene with early-onset type 2 diabetes mellitus in Pima Indians," Thyroid, vol. 22, no. 1, pp. 80-87, 2012.

[13] D. Mentuccia, L. Proietti-Pannunzi, K. Tanner et al., "Association between a novel variant of the human type 2 deiodinase gene Thr92Ala and insulin resistance: evidence of interaction with the Trp64Arg variant of the $\beta$-3-adrenergic receptor," Diabetes, vol. 51, no. 3, pp. 880-883, 2002.

[14] J. M. Dora, W. E. Machado, J. Rheinheimer, D. Crispim, and A. L. Maia, "Association of the type 2 deiodinase Thr92Ala polymorphism with type 2 diabetes: case-control study and meta-analysis," European Journal of Endocrinology, vol. 163, no. 3, pp. 427-434, 2010.

[15] N. Grarup, M. K. Andersen, C. H. Andreasen et al., "Studies of the common DIO2 Thr92Ala polymorphism and metabolic phenotypes in 7342 Danish white subjects," Journal of Clinical Endocrinology and Metabolism, vol. 92, no. 1, pp. 363-366, 2007.

[16] D. Mentuccia, M. J. Thomas, G. Coppotelli et al., "The Thr92Ala deiodinase type 2 (DIO2) variant is not associated with type 2 diabetes or indices of insulin resistance in the Old Order of Amish," Thyroid, vol. 15, no. 11, pp. 1223-1227, 2005.

[17] A. L. Maia, J. Dupuis, A. Manning et al., "The type 2 deiodinase (DIO2) A/G polymorphism is not associated with glycemic traits: the Framingham Heart Study," Thyroid, vol. 17, no. 3, pp. 199-202, 2007. 
[18] J. M. Dora, S. M. Wajner, J. D. Costa et al., “Type 2 deiodinase Thr92Ala polymorphism is associated with disrupted placental activity but not with dysglycemia or adverse gestational outcomes: a genetic association study," Fertility and Sterility, vol. 101, no. 3, pp. 833-839, 2014.

[19] G. Wells, B. Shea, D. O'Connell et al., "The Newcastle-Ottawa Scale (NOS) for assessing the quality of nonrandomised studies in meta-analyses," 2000.

[20] Y. Dhanunjaya, P. B. Dolia, and R. Chitraa, “Type II 5'deiodinase Thr92Ala polymorphism is associated with CVD risk among type 2 diabetes mellitus patients," Journal of Diabetes Mellitus, vol. 6 , no. 1, p. 58, 2015.

[21] A. A. F. Estivalet, L. B. Leiria, J. M. Dora et al., "D2 Thr92Ala and PPAR $\gamma 2$ pro12ala polymorphisms interact in the modulation of insulin resistance in type 2 diabetic patients," Obesity, vol. 19, no. 4, pp. 825-832, 2011.

[22] A. Chidakel, D. Mentuccia, and F. S. Celi, "Peripheral metabolism of thyroid hormone and glucose homeostasis," Thyroid, vol. 15, no. 8, pp. 899-903, 2005.

[23] M. Fiorito, I. Torrente, S. De Cosmo et al., "Interaction of DIO2 T92A and PPAR $\gamma 2$ P12A polymorphisms in the modulation of metabolic syndrome," Obesity, vol. 15 , no. 12, pp. 2889-2895, 2007.

[24] A. L. Maia, S.-J. Hwang, D. Levy, M. G. Larson, P. R. Larsen, and C. S. Fox, "Lack of association between the type 2 deiodinase A/G polymorphism and hypertensive traits: The Framingham Heart Study," Hypertension, vol. 51, no. 4, pp. e22-e23, 2008.

[25] I. Callebaut, C. Curcio-Morelli, J.-P. Mornon et al., "The iodothyronine selenodeiodinases are thioredoxin-fold family proteins containing a glycoside hydrolase clan GH-A-like structure," The Journal of Biological Chemistry, vol. 278, no. 38, pp. 36887-36896, 2003.

[26] Y. Lin and Z. Sun, "Current views on type 2 diabetes," Journal of Endocrinology, vol. 204, no. 1, pp. 1-11, 2010. 


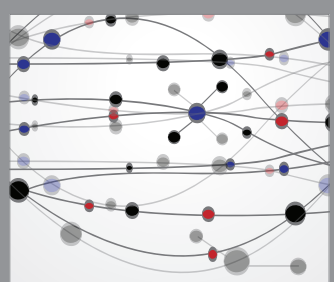

The Scientific World Journal
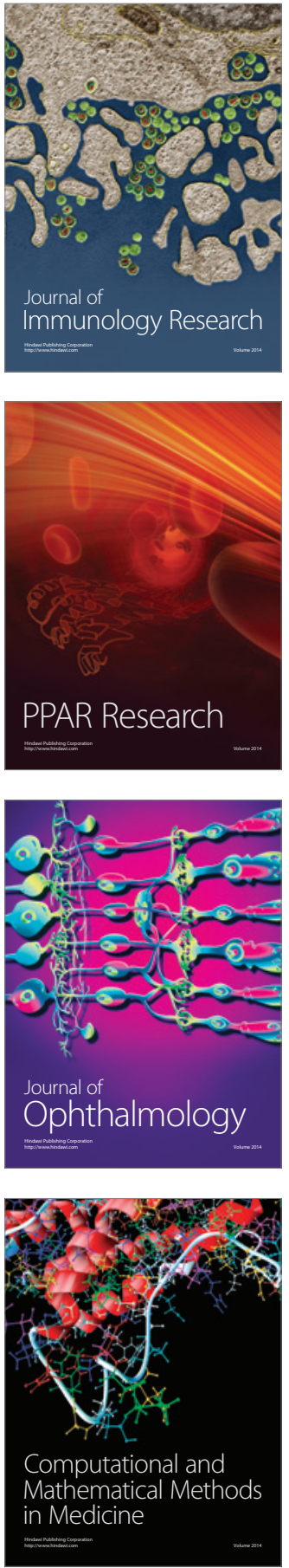

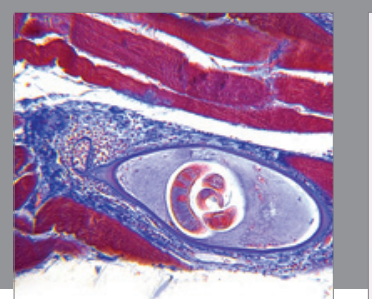

Gastroenterology Research and Practice

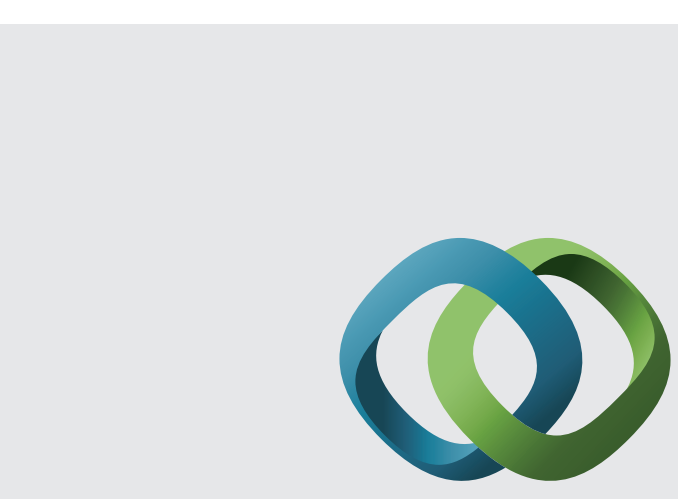

\section{Hindawi}

Submit your manuscripts at

http://www.hindawi.com
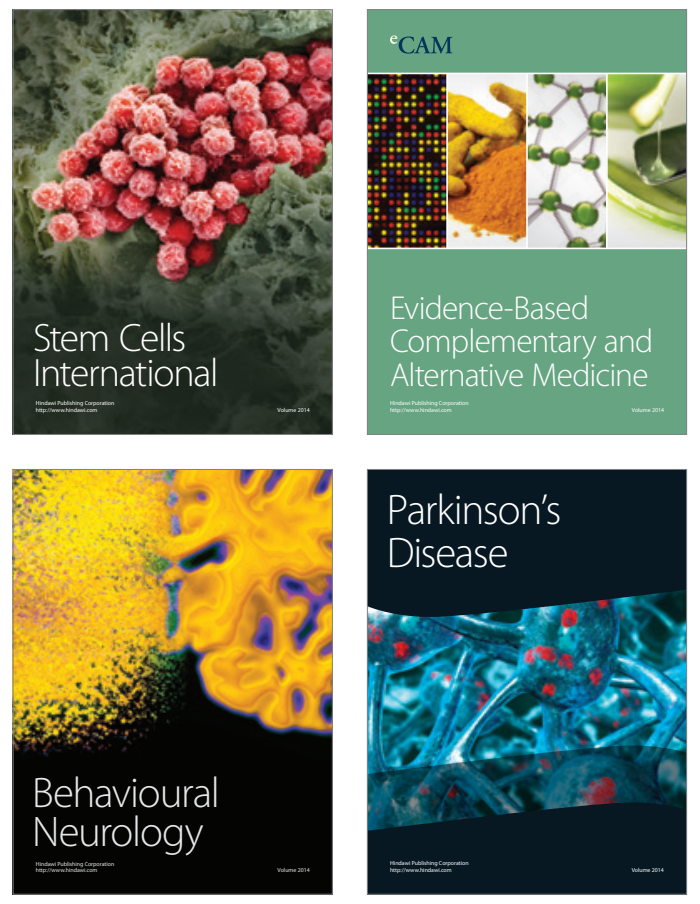
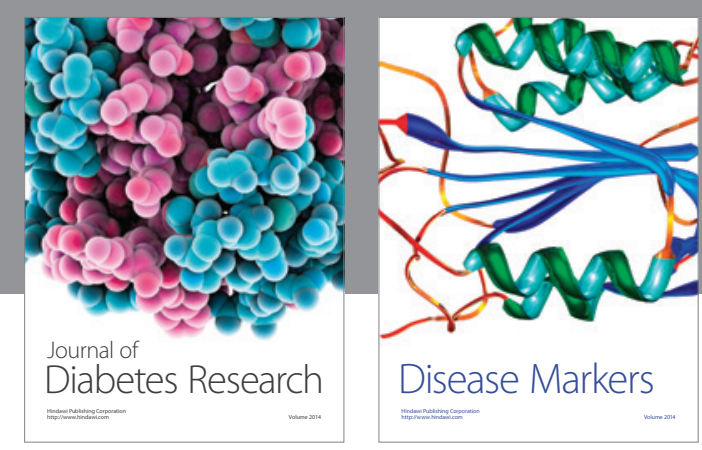

Disease Markers
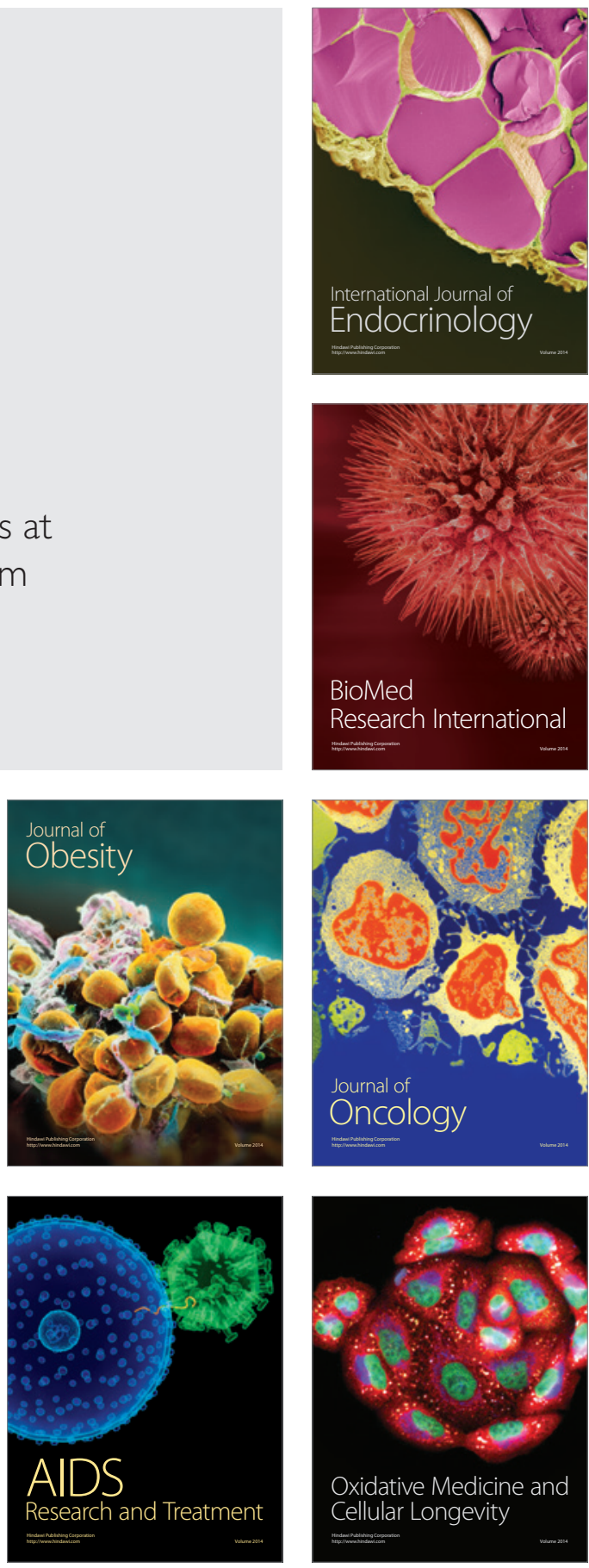